\title{
Online Mass Flow Prediction in CFB Boilers
}

\author{
Andriy Ivannikov ${ }^{1,2}$, Mykola Pechenizkiy ${ }^{1}$, Jorn Bakker ${ }^{1}$, Timo Leino $^{3}$, \\ Mikko Jegoroff ${ }^{3}$, Tommi Kärkkäinen ${ }^{2}$, and Sami Äyrämö ${ }^{2}$ \\ 1 Department of Computer Science, Eindhoven University of Technology, \\ P.O. Box 513, NL-5600 MB, Eindhoven, The Netherlands \\ $\{\mathrm{m}$.pechenizkiy, j.bakker\}@tue.nl \\ 2 Department of Mathematical Information Technology, University of Jyväskylä, \\ P.O. Box 35, FIN-40014, Jyväskylä, Finland \\ aivanni@cc.jyu.fi, \{tka,samiayr\}@mit.jyu.fi \\ 3 VTT, Technical Research Centre of Finland, \\ P.O. Box 1603, FIN-40101, Jyväskylä, Finland \\ \{Timo.Leino, Mikko.Jegoroff\}@vtt.fi
}

\begin{abstract}
Fuel feeding and inhomogeneity of fuel typically cause process fluctuations in the circulating fluidized bed (CFB) process. If control systems fail to compensate for the fluctuations, the whole plant will suffer from fluctuations that are reinforced by the closed-loop controls. This phenomenon causes a reduction of efficiency and lifetime of process components. Therefore, domain experts are interested in developing tools and techniques for getting better understanding of underlying processes and their mutual dependencies in CFB boilers. In this paper we consider an application of data mining technology to the analysis of time series data from a pilot CFB reactor. Namely, we present a rather simple and intuitive approach for online mass flow prediction in CFB boilers. This approach is based on learning and switching regression models. Additionally, noise canceling, and windowing mechanisms are used for improving the robustness of online prediction. We validate our approach with a set of simulation experiments with real data collected from the pilot CFB boiler.
\end{abstract}

\section{Introduction}

Continuous and growing increase of fluctuations in electricity consumption brings new challenges for the control systems of boilers. Conventional power generation will face high demands to ensure the security of energy supply because of increasing share of renewable energy sources like wind and solar power in power production. This can lead to frequent load changes which call for novel control concepts in order to minimize emissions and to sustain high efficiency during load changes.

From the combustion point of view the main challenges for the existing boilers are caused by a wider fuel selection (increasing share of low quality fuels), increasing share of bio fuels, and co-combustion. In steady operation, combustion is affected by the disturbances in the feed-rate of the fuel and by the incomplete

P. Perner (Ed.): ICDM 2009, LNAI 5633, pp. 20€-219, 2009.

(C) Springer-Verlag Berlin Heidelberg 2009 
mixing of the fuel in the bed, which may cause changes in the burning rate, oxygen level, and increase of CO emissions. This is especially important, when considering the new biomass based fuels, which have increasingly been used to replace coal. These new biofuels are often rather inhomogeneous, which can cause instabilities in the feeding. These fuels are usually also very reactive. Biomass fuels have much higher reactivity compared to coals and the knowledge of the factors affecting the combustion dynamics is important for optimum control. The knowledge of the dynamics of combustion is also important for optimizing load changes [9].

Different needs for facilitating intelligent analysis of time series data from CFB sensors measurements, which would lead to better understanding of underlying processes in the $\mathrm{CFB}$ reactor, were considered in 6. In general, a data-mining approach can be used to develop a model for optimizing the efficiency of an CFB boiler. E.g. the selection of process variables to optimize combustion efficiency has been studied in 10. Another typical problem, which we address in this work, is online reliable prediction of system parameters which can not be measured reliably in a direct way.

In this paper we focus on the problem of online mass flow prediction. This is an important problem since knowing mass flow is necessary for the control optimization, but measuring it directly in the CFB operational settings is difficult. Currently, the mass flow is calculated offline using relatively long time period averages. We propose a rather simple and intuitive approach for online mass flow prediction, which is based on learning and switching regression models. We validate our ideas with a set of simulation experiments with real data collected from the pilot CFB boiler.

The rest of the paper is organized as follows. Section 2 present a wider context of sensor data mining for developing better understanding and control of CFB reactors. In Section 3 we discuss the problem of obtaining mass flow signal from the CFB pilot boiler. In Section 4 we outline our approach for online mass flow prediction. The experimental results are discussed in Sections 5. We briefly conclude with a summary and discussion of the further work in Section 6 .

\section{A Data Mining Approach for CFB Understanding and Control}

The supercritical CFB combustion utilizes more cleanly, efficiently, and sustainable way coal, biofuels, and multifuels, but need advanced automation and control systems because of their physical peculiarities (relatively small steam volume and absence of a steam drum). Also the fact that fuel, air, and water mass flows are directly proportional to the power output of the boiler sets tight demands for the control system especially in CFB operation where huge amount of solid material exist in the furnace.

When the CFB boilers are becoming larger, not only the mechanical designs but also the understanding of the process and the process conditions affecting heat transfer, flow dynamics, carbon burnout, hydraulic flows etc. have been 
important factors. Regarding the furnace performance, the larger size increases the horizontal dimensions in the CFB furnace causing concerns on ineffective mixing of combustion air, fuel, and sorbent. Consequently, new approaches and tools are needed in developing and optimizing the CFB technology considering emissions, combustion process, and furnace scale-up.

Fluidization phenomenon is the heart of CFB combustion and for that reason pressure fluctuations in fluidized beds have been widely studied during last decades. Other measurements have not been studied so widely. Underlying the challenging objectives laid down for the CFB boiler development it is important to extract as much as possible information on prevailing process conditions to apply optimization of boiler performance. Instead of individual measurements combination of information from different measurements and their interactions will provide a possibility to deepen the understanding of the process.

A very simplified view on how a CFB boiler operates is presented in the upper part of Fig. 1. Fuel (mixture of fuels), air, and limestone are the controlled inputs to the furnace. Fuel is utilized to heat production; air is added for enhancing the combustion process and limestone is aimed at reducing the sulfur

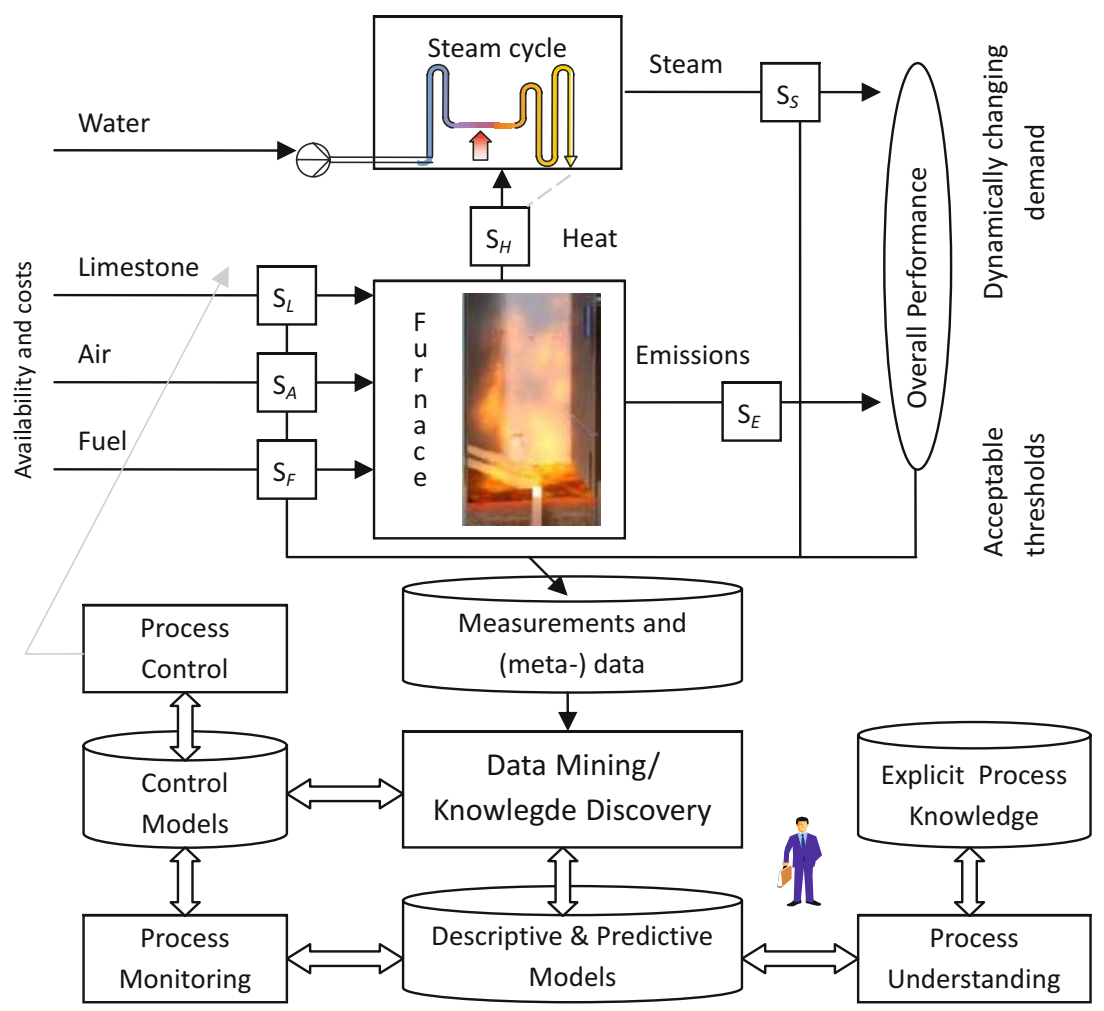

Fig. 1. A simplified view of a CFB boiler operation with the data mining approach 
dioxides (SO2). The produced heat converts water into steam that can be utilized for different purposes. The measurements from sensors SF, SA, SL, SH, SS and SE that correspond to different input and output parameters are collected in database repository together with other meta-data describing process conditions for both offline and online analysis. Conducting experiments with pilot CFB reactor and collecting their results into database creates the necessary prerequisites for utilization of the vast amount of DM techniques aimed to identifying valid, novel, potentially useful, and ultimately understandable patterns in data that can be further utilized to facilitate process monitoring, process understanding, and process control.

\section{The Problem of Obtaining Mass Flow Signal}

The combustion and emission performance of different types of solid fuels and their mixtures are studied at VTT's $50 \mathrm{~kW}$ CFB pilot boiler (Fig. 2). The height of the riser of the boiler is $8 \mathrm{~m}$ and the inner diameter $167 \mathrm{~mm}$. The reactor is equipped with several separately controlled electrically heated and water/air cooled zones in order to control the process conditions, for example, oxygen level, temperature and load almost independently. Several ports for gas and solid material sampling are located in the freeboard area.
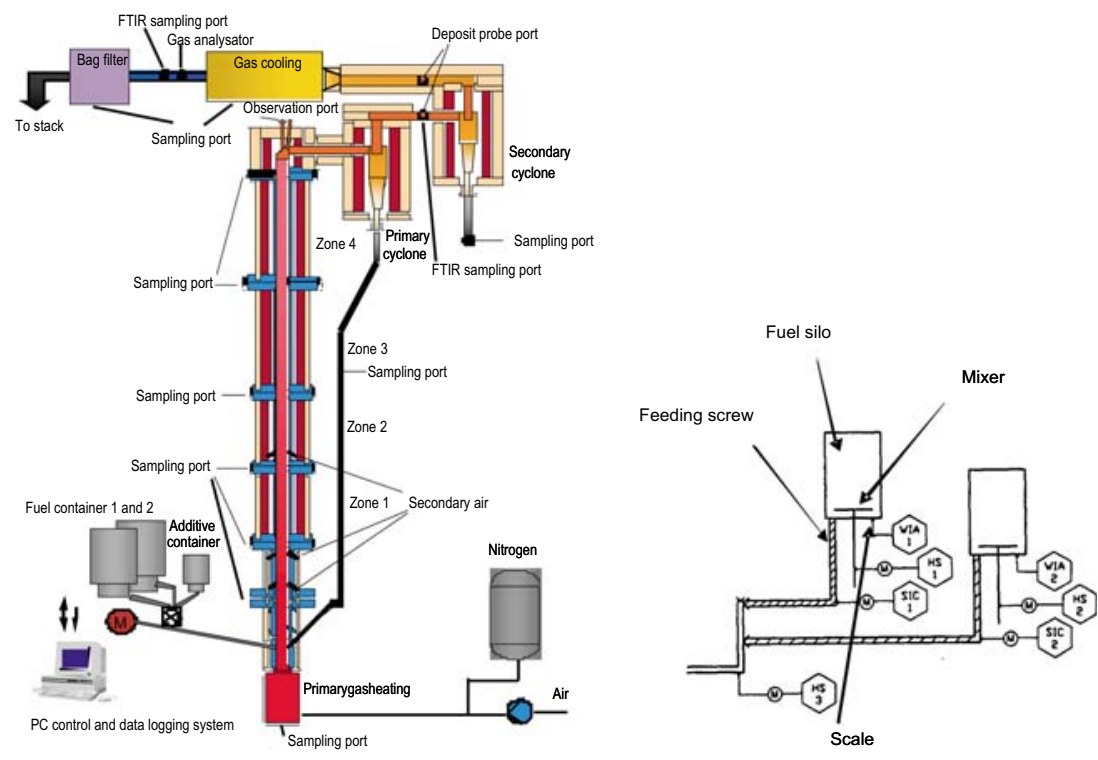

Fig. 2. A schematic diagram of the laboratory scale CFB-reactor (left) and its fuel feeding system (right) 
The fuel can be fed into the reactor through two separate fuel feeding lines (Fig. 2). In each line there is a fuel screw feeder on the bottom of the silo and also a mixer which prevents arching of the fuel in the silo. The fuel silos are mounted on the top of scales which enables the determination of mass flow rates for solid fuels as a weight loss against time. Some of the phenomena that make the scale signal fluctuate with constant screw feeder rotational speed are:

- the quality of the fuel changes (e.g. moisture content, particle size);

- the fuel grades in the silo;

- some of the particles jam in between the screw rotor and stator causing a peak in the mass signal;

- fuel addition causes a step change in the signal

The amount of the fuel in the silo has an effect on the mass flow. This causes that mass flow decreases in between fillings as the level of fuel in the tank decreases

Due to the fluctuation in the scale signal no reliable online data can be obtained from the mass flow of fuel to the boiler. The measurement system cannot be easily improved and therefore the mass flow is calculated offline using longer time period averages. The aim of the study was to remove the problematic fluctuation and fuel filling peaks from the data using signal processing methods in order to obtain online mass flow signal which could be used among others for mass flow control of fuel at the CFB-pilot.

\section{Our Approach: Context-Sensitive Learning}

In case of CFB boiler (like in many other dynamic environments) the data flows continuously and the target concept (that is the mass flow in this study) could change over time due to the different operational processes (like fuel loading) or changes in these processes themselves. Therefore, learning algorithms must recognize abrupt (in case of mass flow) change in the target concept and adjust a model accordingly.

For this study, it is assumed that the transitions from fuel feeding processes to fuel consuming processes are known. The focus will be on a learning algorithm that is able to track the mass flow given the high frequency nature of the signal and the changing fuel types for each of the processes.

In this section we consider several practical aspect, including the use of domain knowledge and experimental settings metadata, and noise toleration in online mass flow prediction.

\subsection{Integration of Domain Knowledge and Experimental Settings Metadata}

The overall measurements during the experiment are presented in Fig. 3.

The data was recorded with $1 \mathrm{~Hz}$ sampling rate. In each test the type of fuel and/or the rpm of the feeding screw were varied. The rotation of the mixing 


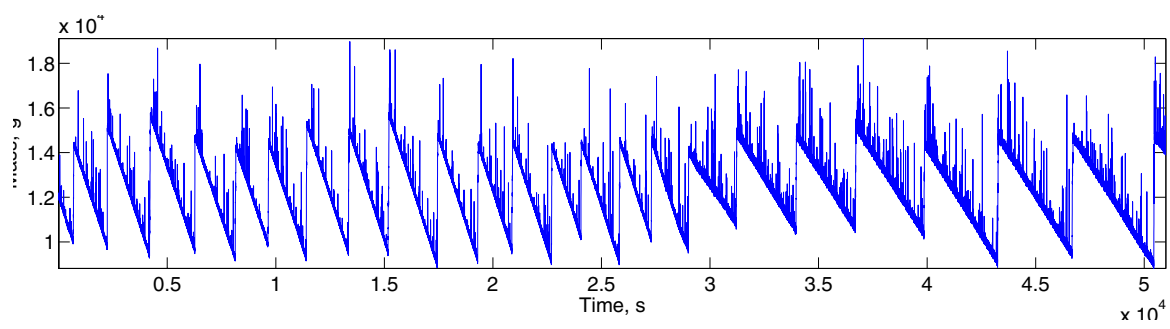

Fig. 3. Measurements of the fuel mass in the tank

screw was kept constant at $5.5 \mathrm{rpm}$. The three major sources of noise in the measurements are mixing and feeding screws and the occasional jamming of the fuel particle between the stator and rotor in the screw. The rotation of the screws causes vibrations to the system that are reflected by the scales as high frequency fluctuations around the true mass value. In Fig. 4 the evolution of the frequency content of the measurements is shown by means of the short-time Fourier transform [5], from which the influence of the screws is evident. Namely, the rotating parts induce oscillations to the measurements of the same frequency as the rotation frequency. The frequency content due to the screws is identified from the figure as contrasting vertical curves.

The jamming of the fuel particle causes an abnormally large upward peak to the measurements that can be seen from Fig. 3. The speed of the mass change in the tank at a given time depends not only on the rpm of the feeding screw and the speed of the replenishment of the fuel in the tank, but also on the amount of the fuel in the tank. The more fuel is in the tank the more fuel gets in the screw, since the weight of the fuel at the higher levels of the tank compresses (increases the density) the fuel in the lower levels and in the screw. The size and grade of fuel also have an effect on the compression rate of the fuel. Therefore, we assume that the mass flow signal has nonzero second derivative. Thus the nature of the phenomena measured by the scales can be modeled using the following equation:

$$
\begin{aligned}
& y\left(t-t_{0}\right)=\frac{a \cdot\left(t-t_{0}\right)^{2}}{2}+v_{0} \cdot\left(t-t_{0}\right)+ \\
& m_{0}+A \cdot \sin \left(\omega_{f e e d} \cdot\left(t-t_{0}\right)+\alpha_{f e e d}\right)+ \\
& B \cdot \sin \left(\omega_{\text {mix }} \cdot\left(t-t_{0}\right)+\alpha_{\text {mix }}\right)+e\left(t-t_{0}\right),
\end{aligned}
$$

where $y\left(t-t_{0}\right)$ denotes the time series of the output of the scales at time $t-t_{0}, a$ is acceleration of the mass change, $v_{0}$ stands for the speed of the mass change at time $t_{0}, m_{0}$ is the initial mass at time $t_{0} ; A$ and $B, \omega_{\text {feed }}$ and $\omega_{m i x}, \alpha_{\text {feed }}$ and $\alpha_{m i x}$ are amplitude, frequency and phase of the fluctuations caused by feeding and mixing screws, respectively; $e\left(t-t_{0}\right)$ denotes the random peaked high amplitude noise caused by the jamming of the fuel particle at time $t-t_{0}$. 


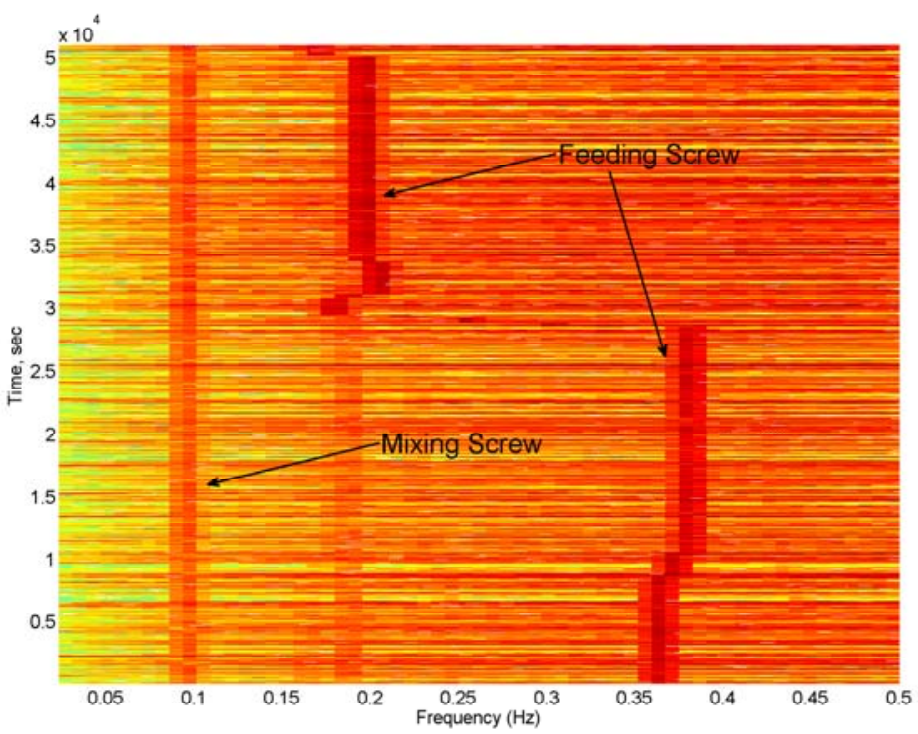

Fig. 4. Spectrogram of the fuel mass measurements computed using Short-Time Fourier Transform. The color denotes the power spectral density (PSD).

Formally, the problem of denoising/approximating the true mass flow signal consists in extracting the component related to the mass flow from the measured time series:

$$
m\left(t-t_{0}\right)=\frac{a \cdot\left(t-t_{0}\right)^{2}}{2}+v_{0} \cdot\left(t-t_{0}\right)+m_{0},
$$

where $m\left(t-t_{0}\right)$ denotes the value of the mass at time $t-t_{0}$. One solution to this problem is to use stochastic gradient descent [1] to fit the model (1) without $e\left(t-t_{0}\right)$ term to the measured data with the high amplitude peaks skipped from the learning process. This is closely related to fitting the model (2) to the same data in the mean-least-squares sense as the noise fluctuations are symmetric relatively to the true mass signal. Alternatively a linear regression approach with respect to the second order polynomial can offer a better local stability and faster convergence. As the accurate mass flow measurements are required on-line by a control system the choice of the linear regression method seems more reasonable.

The linear regression can be performed by using the Vandermonde matrix [3], whose elements are powers of independent variable $x$. In our case the independent variable is time $x_{i}=t_{i-1}-t_{0}, i=1, \ldots, T$, where $T$ denotes the number of the time samples. If the linear regression is done for a polynomial of order $n$ $\left(p^{n}(x)=p_{n} x^{n}+p_{n-1} x^{n-1}+\ldots+p_{1} x+p_{0}\right)$ the Vandermonde matrix is computed from the observed time series of the independent variable as follows:

$$
v_{i, j}=x_{i}^{n-j+1}, i=1, \ldots, T, j=1, \ldots, n+1,
$$


where $i$ and $j$ run over all time samples and powers, respectively. Provided the Vandermonde matrix the problem of polynomial interpolation is solved by solving the system of linear equations $\mathbf{V} \mathbf{p} \cong \mathbf{y}$ with respect to $\mathbf{p}$ in the least square sense:

$$
\widehat{\mathbf{p}}=\operatorname{argmin}_{\mathbf{p}} \sum_{i=1}^{T}\left(\sum_{j=1}^{n+1} V_{i, j} p_{n-j+1}-y_{i}\right)^{2}
$$

Here, $\mathbf{p}=\left[\begin{array}{lllll}p_{n} & p_{n-1} & \ldots & p_{1} & p_{0}\end{array}\right]^{\mathrm{T}}$ denotes the vector of the coefficients of the polynomial, and $\mathbf{y}=\left[y\left(x_{1}\right) y\left(x_{2}\right) \ldots y\left(x_{T}\right)\right]^{\mathrm{T}}=\left[\begin{array}{lll}y_{1} & y_{2} \ldots y_{T}\end{array}\right]^{\mathrm{T}}$ is the time series of the dependent variable that is indication of the scales. Provided that the $n+1$ columns of the matrix $\mathbf{V}$ are linearly independent, this minimization problem has a unique solution given by solving the normal equation [4]:

$$
\left(\mathbf{V}^{\mathrm{T}} \mathbf{V}\right) \widehat{\mathbf{p}}=\mathbf{V}^{\mathrm{T}} \mathbf{y}
$$

\subsection{Application}

In this section we consider practical aspects of application of the least square approximation to our case study. For the modeling of the mass flow we have chosen second order polynomial. The estimated coefficients of the polynomial have the following meanings: $\widehat{p}_{0}=\widehat{m}_{0}, \widehat{p}_{1}=\widehat{v}_{0}, \widehat{p}_{2}=\widehat{a}$. We distinguish the two types of the periods in the experiment: the consumption (fuel is only consumed) and the consumption with fuel replenishment. When one period of the CFB operating changes to another (i.e. a new portion of fuel is being added) the process of mass flow approximation and prediction starts over again, as the model of the mass flow changes. Thus, the most problematic unstable regions are the transitions intervals, when the parameters of the model change their values. First, we describe the on-line approach and then move to a semi-on-line one, which offers a more accurate estimates of the mass flow parameters in the beginning of the period. When a period of the session starts the samples of measurements start to accumulate in the buffer. The data in the buffer are used to approximate the mass flow signal, i.e., to fix the parameters of the approximation/interpolation model. Only the samples that do not contain high amplitude peak due to jamming are placed to the buffer. As the amplitude of these peaks is abnormally high, they can easily be detected on-line as exceeding a certain threshold that should be higher for the replenishment period. The time when a period changes to another is precisely known as it is manipulated by the system operator. At these times the buffer is emptied and starts to accumulate new data. The first measurement within the experiment is taken as the approximation of the mass flow signal at that time $\widehat{m}_{1}=y_{1}$ and as the first point that is placed to the buffer. In contrast, the first approximation of the mass within a following period is taken as the last approximation of the mass from the previous period that is obvious. In addition the last approximation of the mass from the previous period $\widehat{m}_{i_{c}}$ is placed to the buffer as the first point for the new period, where $i_{c}$ denotes the number 
of the sample when the change of the periods occurs. When a new sample arrives the parameters of the model are estimated based on the points that are in the buffer independently of whether the current point was placed to the buffer or not. The current approximation of the mass signal is computed based on the current model. Depending on the number of data points in the buffer different approximation models apply:

1. If the number of points in the buffer amounts to one, then the current approximation of the mass is taken as the approximation of the mass at previous iteration $\widehat{m}_{i}=\widehat{m}_{i-1}$.

2. If the number of points in the buffer is $1<T \leq 4$, then $\widehat{p}_{2}$ is set to zero and the parameters $\widehat{p}_{0}$ and $\widehat{p}_{1}$ of the first order polynomial are estimated from the available data. The approximation of the mass at the current time sample $x_{i}$ is taken as $\widehat{m}_{i}=\widehat{p}^{1}\left(x_{i}\right)$, where $\widehat{p}^{1}(x)$ is the current approximation of mass flow model by the first order polynomial.

3. If the number of points in the buffer is larger than four then the second order polynomial model is fitted to the data, and the current approximation of the mass is computed as $\widehat{m}_{i}=\widehat{p}^{2}\left(x_{i}\right)$, where $\widehat{p}^{2}(x)$ is the current approximation of mass flow model by the second order polynomial.

In practice, the operational settings often allow a delay between the data arrival and the evaluation of the signal of interest at this time sample. This means that the estimate of the signal at a given time sample is obtained based on the data that are accumulated also during the future times. This allows the more accurate estimates of the signal to be computed. Note that in our case this will have an effect of increased accuracy mainly for the beginning of the period, when the amount of the data in the buffer is small yet. The extension of the previous considerations to a case with a certain delay time is straightforward: the estimation of the mass signal is delayed in respect to the current time and the filling of the buffer by the delay $\tau$ expressed in time sample units. A minor concern is related to the last time points within a period, for which the specified delay can not be applied as the end of the period is reached. As we already mentioned, when the amount of accumulated data is large the influence of the time delay becomes insignificant in respect to the estimate of the signal. Thus, the last approximations of the mass within the period can be obtained without a delay. Alternatively, if the delay $\tau$ is used, for the last $\tau$ approximations of the mass within the period the following rule can be used $\widehat{m}_{i_{c}-\tau+k}^{\tau}=\widehat{m}_{i_{c}-\tau+k}^{\tau-k}, k=1, \ldots, \tau$. Here, $\widehat{m}_{i}^{\tau}$ denotes the estimate of the mass at time sample $i$ using the delay $\tau$. The latter means that the last approximations of the mass signal for delay $\tau$ are taken as approximations of the signal from the approaches with smaller delay. This option can be useful for the replenishment period, which is usually short, as even at the end of this period the amount of data in the buffer is small. In this case it is important to use as large delay as possible, to obtain smoother and more accurate signal approximation. 


\section{$5 \quad$ Experimental Results}

As the main points of interest are the points, where the change of the period occurs, for the analysis we took an interval of the experiment containing both types of the transition points (see Fig. (5)).

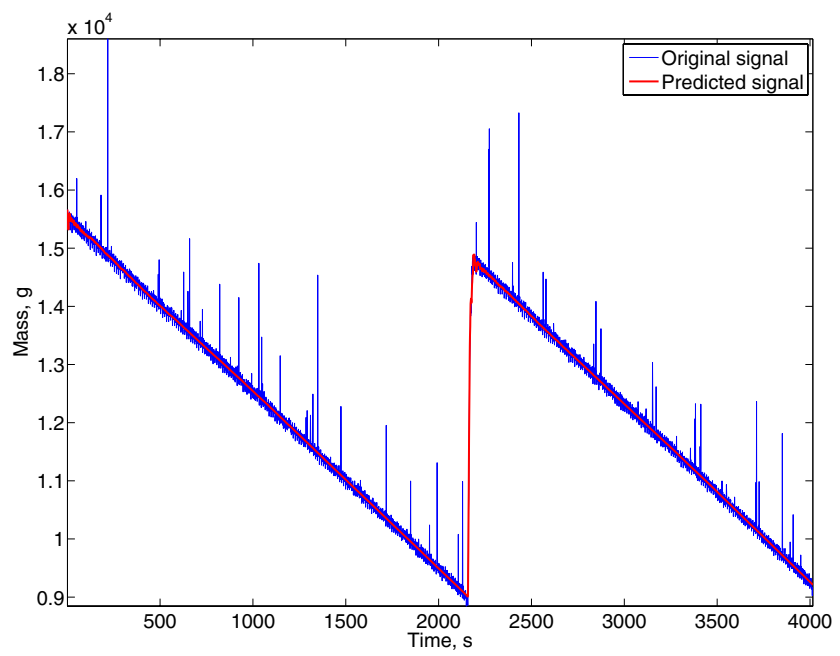

Fig. 5. Online mass flow prediction with the switching regression models and zero delay time
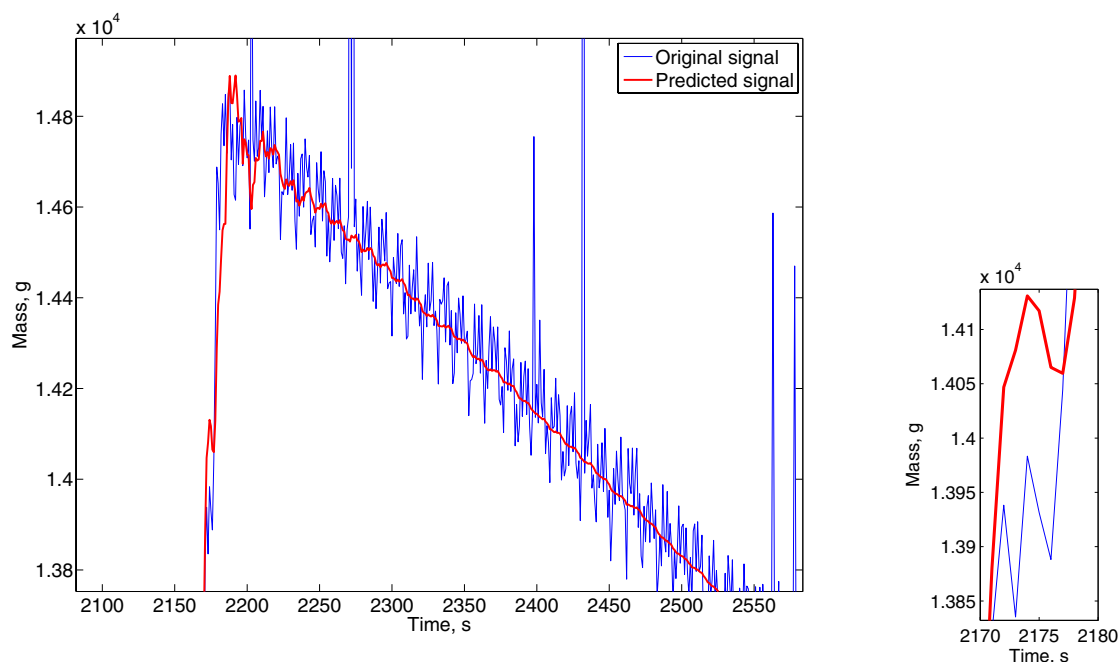

Fig. 6. Online mass flow prediction with the switching regression models and zero delay time. Zooming to transition point. 


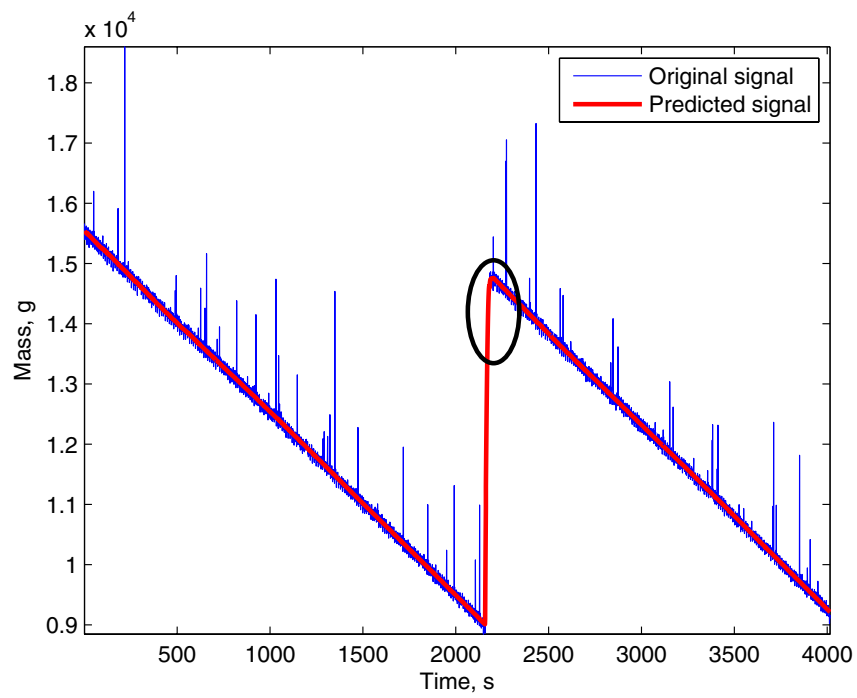

Fig. 7. Online mass flow prediction with the switching regression models and 20 samples delay time
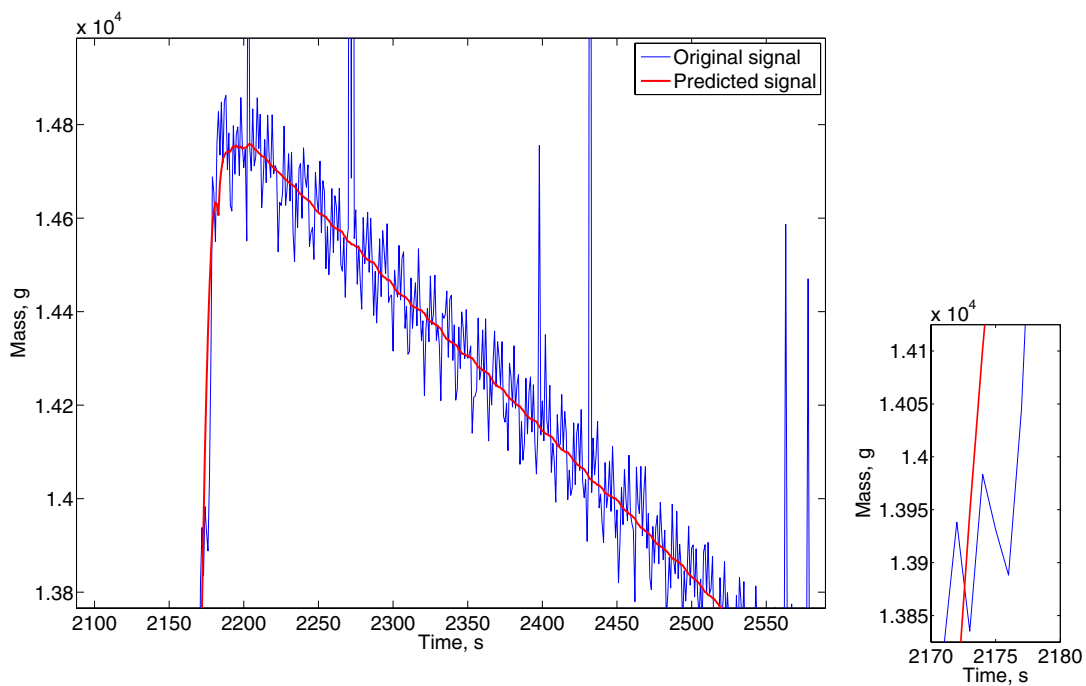

Fig. 8. Online mass flow prediction with the switching regression models and 20 samples delay time. Zooming to transition point.

The computations of mass denoising were done for the delay time varying from 0 to 20 samples/seconds with step 1 . In Fig. 5 and 6 the resulting approximation of mass for zero delay is shown in different scales. 
It takes about $100-150$ seconds from the beginning of each consumption period for the model of the mass signal stabilizes. Since the replenishment period lasts during shorter times $(3-40$ seconds), the model may exhibit instability even at the end of the period. However, overall the approximation of the mass during the replenishment period is satisfactory, because changes of the mass are rather steep and prominent against the background of the noise. For comparison, the effect of increased delay time is shown in Fig. 7 and 8, where the delay time 20 seconds was used.

With this delay time the model is relatively well stabilized already in the beginning of each period, which offers clear accuracy advantage. To indicate the rate of convergence of the model with respect to the delay time, we computed the mean-square error between the mass signal approximations for each pair of the consecutive delays (see Fig. 9):

$$
\operatorname{MSE}(\tau)=\frac{1}{T} \sum_{i=1}^{T}\left(\widehat{m}_{i}^{\tau}-\widehat{m}_{i}^{\tau-1}\right)^{2} .
$$

It can be clearly seen from the figure that for the small delays ( $1-4$ samples) the approximation of the signal improves dramatically, and for the larger delays the improvement slows down.

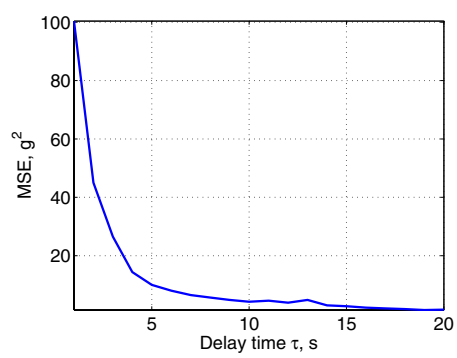

Fig. 9. Mean-square error between the mass signal approximations for each pair of the successive delays

\section{Conclusions and Further Work}

Prediction of mass flow in CFB boilers in online settings is an important and challenging problem having some connections to the problem of learning under the presence of sudden concept drifts.

In this paper we presented a rather simple regression learning approach with filtering of outliers and the dynamic switching of predictors depending on the current state of the fuel feeding/reloading process that was assumed to be known.

Our experimental study demonstrated that this approach performs reasonably well. A delay of less than 5 seconds allows to predict the mass flow accurately enough to be used as a reliable indicator for the CFB control system. 
The directions of our further work in mining CFB sensor data include (1) studying the effect of fuel feeding speed, and the effect of using different mixtures of fuels, and (2) external validation of our online mass flow prediction approach being implemented as part of the control system of the pilot CFB boiler in operational settings with different conditions. We anticipate that local reliability estimation of mass flow prediction [7] may be helpful for the domain experts. We also plan to adopt and develop further the dynamic integration of regression models [8] which may help to improve the reliability of predictions.

Currently the implementation assumes that the transitions between fuel feeding and fuel consumption phases are known. However, to adapt the outlier removal and prediction procedures to more general settings, when the knowledge about the state changes is not available, the method for automatic on-line identification of the state changes would be of benefit.

Hence, automating the predictions of the mass flow by developing an algorithm that is able to detect these state transitions is one of the next steps. In data mining and machine learning these changes are generally known as concept drift, that is the changes in the (hidden) context inducing more or less radical changes in the target concept [11. The challenge is to keep track of the drift and adjust the model accordingly. This might be possible by using statistics of the performance of the model [2] or by keeping a set of models and select the best. And all of this needs to be done by an online method.

\section{Acknowledgements}

This research is partly supported by TEKES (Finnish Funding Agency for Technology and Innovations) DYNERGIA project and NWO (The Netherlands Organization for Scientific Research) HaCDAIS project.

\section{References}

1. Duda, R.O., Hart, P.E., Stork, D.G.: Pattern Classification, 2nd edn. Wiley Interscience, Hoboken (2001)

2. Gama, J., Castillo, G.: Learning with local drift detection. In: Li, X., Zaïane, O.R., Li, Z.-h. (eds.) ADMA 2006. LNCS, vol. 4093, pp. 42-55. Springer, Heidelberg (2006)

3. Horn, R.A., Johnson, C.R.: Topics in matrix analysis. Cambridge University Press, Cambridge (1991)

4. Lawson, C.L., Hanson, R.J.: Solving Least Squares Problems. Prentice-Hall, Englewood Cliffs (1974)

5. Nawab, S., Quatieri, T.: Short time fourier transform. In: Lim, J., Oppenheim, A. (eds.) Advanced topics in signal processing, pp. 289-337. Prentice-Hall, Englewood Cliffs (1988)

6. Pechenizkiy, M., Tourunen, A., Kärkkäinen, T., Ivannikov, A., Nevalainen, H.: Towards better understanding of circulating fluidized bed boilers: a data mining approach. In: Proceedings ECML/PKDD Workshop on Practical Data Mining, pp. 80-83 (2006) 
7. Rodrigues, P.P., Gama, J., Bosnic, Z.: Online reliability estimates for individual predictions in data streams. In: ICDM Workshops, pp. 36-45. IEEE Computer Society Press, Los Alamitos (2008)

8. Rooney, N., Patterson, D.W., Anand, S.S., Tsymbal, A.: Dynamic integration of regression models. In: Roli, F., Kittler, J., Windeatt, T. (eds.) MCS 2004. LNCS, vol. 3077, pp. 164-173. Springer, Heidelberg (2004)

9. Saastamoinen, J.: Modelling of dynamics of combustion of biomass in fluidized beds. Thermal Science 8(2), 107-126 (2004)

10. Song, Z., Kusiak, A.: Constraint-based control of boiler efficiency: A data-mining approach. IEEE Trans. Industrial Informatics 3(1), 73-83 (2007)

11. Widmer, G., Kubat, M.: Learning in the presence of concept drift and hidden contexts. Mach. Learn. 23(1), 69-101 (1996) 